\title{
Investigation of Fluidic Devices for Mixing Enhancement for the Shockless Explosion Combustion Process
}

\author{
Bernhard C. Bobusch ${ }^{1}$, Phillip Berndt ${ }^{2}$ Christian Oliver Paschereit $^{1}$, and \\ Rupert Klein ${ }^{2}$ \\ 1 Technische Universität Berlin, Institut für Strömungsmechanik und Ttechnische \\ Akustik, - Hermann-Föttinger-Institut - \\ Müller-Breslau-Str. 8; D-10623 Berlin, Germany, \\ bernhard. bobusch, oliver. paschereit@tu-berlin.de \\ ${ }^{2}$ Freie Universität Berlin, Department of Mathematics, Geophysical Fluid Dynamics, \\ Arnimallee 6; D-14195 Berlin, Germany, \\ pberndt, rupert.klein@math.fu-berlin.de
}

\begin{abstract}
Fuel-air mixing is a crucial process in low emission combustion systems. A higher mixing quality leads to lower emissions and higher combustion efficiencies. Especially for the innovative constant volume combustion processes "Shockless Explosion Combustion" (SEC) the mixing of fuel and air is an important parameter, since the whole combustion process is triggered and controlled via the equivalence ratio. To enhance the passive scalar mixing, fluidic oscillators are investigated and compared to the standard jet in crossflow fuel injection configurations. The mixing quality of the different geometries is assessed in a water test-rig by making use of planar laser induced fluorescence. After a short introduction to the SEC-process, the test-rig and the different injection configurations are introduced. To verify whether the mixing quality is sufficient for the SEC-process, a numerical investigation using the experimentally determined unmixedness is conducted. It is not only shown that the fluidic oscillators are able to enhance the mixing quality and create an independence of the mixing quality from the jet in crossflow momentum, but it is also verified in a first numerical calculation that the achieved mixing quality might be good enough for the Shockless Explosion Combustion process.
\end{abstract}

Keywords: mixing $\cdot$ constant volume combustion $\cdot$ Shockless Explosion Combustion $\cdot$ numerical combustion

\section{Introduction}

High quality mixing is one of the most crucial parameters in modern low emission combustion systems. This becomes even more crucial regarding advanced homogeneous pulsed (constant volume) combustion approaches such as the homogeneous charge compression ignition (HCCI) for internal combustion engines 


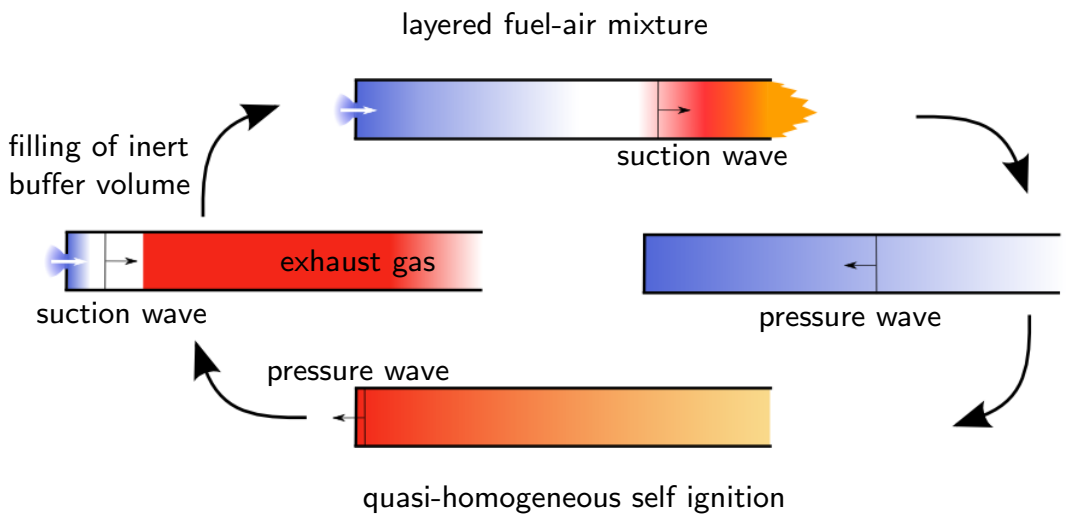

Fig. 1: Process cycle of the shockless explosion combustor

or the shockless explosion combustion (SEC) process for gas turbine combustion. Both of these systems rely on the auto-ignition of a homogeneous fuel-air-charge inside a combustor, thus, the preparation of a perfectly mixed combustion charge is the most important factor for these systems.

For the SEC-process not only is a perfectly homogeneous fuel-air mixture needed, but in addition, a stratification of the equivalence ratio in the combustor is necessary. An overview of this process is shown in Fig. 1. Like other constant volume combustion processes, the shockless explosion combustion process is based on a periodic combustion process. A standing pressure wave is established inside the combustion tube. The moment this pressure wave reduces the pressure at the tube inlet below the plenum pressure, the tube is filled with compressor air (left). After filling a volume with pure air, fuel is added to the combustion air at the inlet position until around $40 \%$ of the tube is filled with a combustible mixture (top). The air volume is needed to separate the hot flue gases of the previous cycle from the fresh fuel-air mixture. The suction wave is reflected from the open end of the tube and travels upstream to the inlet (right). Due to the hot air from the compressor, the mixture undergoes auto-ignition (bottom). The equivalence ratio inside the combustion chamber is adjusted in a way that the ignition delay matches the residence time of the mixture in the tube. Thus, the entire fuel-air volume undergoes homogeneous auto-ignition and the mixture is burnt instantaneously without any shock waves. In addition, the ignition delay is adjusted to match the oscillation period. This means that the combustion of the mixture occurs simultaneously with the pressure wave raising the pressure at the tube inlet. The pressure wave is amplified and travels to the end of the combustion tube, where it is reflected as a suction wave and restarts the process.

This process has by implication very high demands on the mixing quality:

1. The mixing needs to be very fast to avoid regions where the ignition delay is too short. 
2. The mixing must be homogeneous in the radial plane.

3. The axial stratification of equivalence ratio demands for very low mixing in the axial direction.

4. The mixing must be independent from the fuel volume flow (i.e., jet in crossflow momentum) to have a broad range of possible equivalence ratios for base load, part load, and idle operation.

To create this type of mixing several different jet in crossflow configurations are investigated in this work. Namely the tested configurations are round jet, rectangular jet, slit, and spatially oscillating jet in crossflow. The jet in crossflow configuration is the easiest, most robust, and very common way to mix two fluids. Over the past decades, the round turbulent jet in crossflow was widely investigated as a steady jet (e.g., [1-4]) or as a modulated/pulsed jet (e.g., [5-7]). The rectangular jet in crossflow is not as thoroughly described as the round jet, but recently this configuration was analyzed more extensive as well (see $[8,9]$ ). The slit injection lies somewhere between these two configurations. It can be seen as a two-dimensional jet in crossflow with infinite depth. For the spatially oscillating jet, only a few publications can be found. Nathan et al. [10] extensively investigated jet parameters and their impact on the mixing for an inline configuration. Several oscillating devices, mechanical and fluidic, were tested. It was shown that the oscillation enhances the mixing especially close to the fuel inlet. Arnaud and Paschereit [11] tested the enhancement of scalar mixing due to a spatial oscillation of a jet in crossflow in a water test-rig. They confirmed the findings of Nathan et al. [10] and in addition found out that the spatial oscillation reduces the dependency of the mixing quality on the jet in crossflow momentum. These advantages make the spatially oscillating jet in crossflow injection very promising for the demands of the shockless explosion combustion process. To assess and compare the mixing quality of the different configurations they were investigated in a water test-rig making use of a fluorescent dye.

Known from literature (e.g., $[12,13])$, the mixing of water with dye is a good indicator for the fuel-air mixing in combustion systems. In addition, the results of the experiments are used in a numerical calculation to see if the mixing quality is high enough to assure a reliable SEC-process.

The remainder of the paper is organized as follows: First the experimental setup, measurement technique, and evaluation methods are presented. This is followed by the experimental results and the numerical investigations.

\section{Experimental Setup}

To investigate the mixing performance of several geometries, a test-rig was build employing water as the fluid. The entire geometry is based on the planned SEC combustion tube. A schematic overview of the rig can be found in Fig. 2. A more detailed cut through the dye inlet section can be found in Fig. 3. The test-rig consists of a main combustion tube made of acrylic glass with an inner diameter of $40 \mathrm{~mm}$ and a length of $800 \mathrm{~mm}$. Upstream of this tube a $25 \mathrm{~mm}$ valve is installed to open and close the main mass flow. It is followed by a diffuser which 


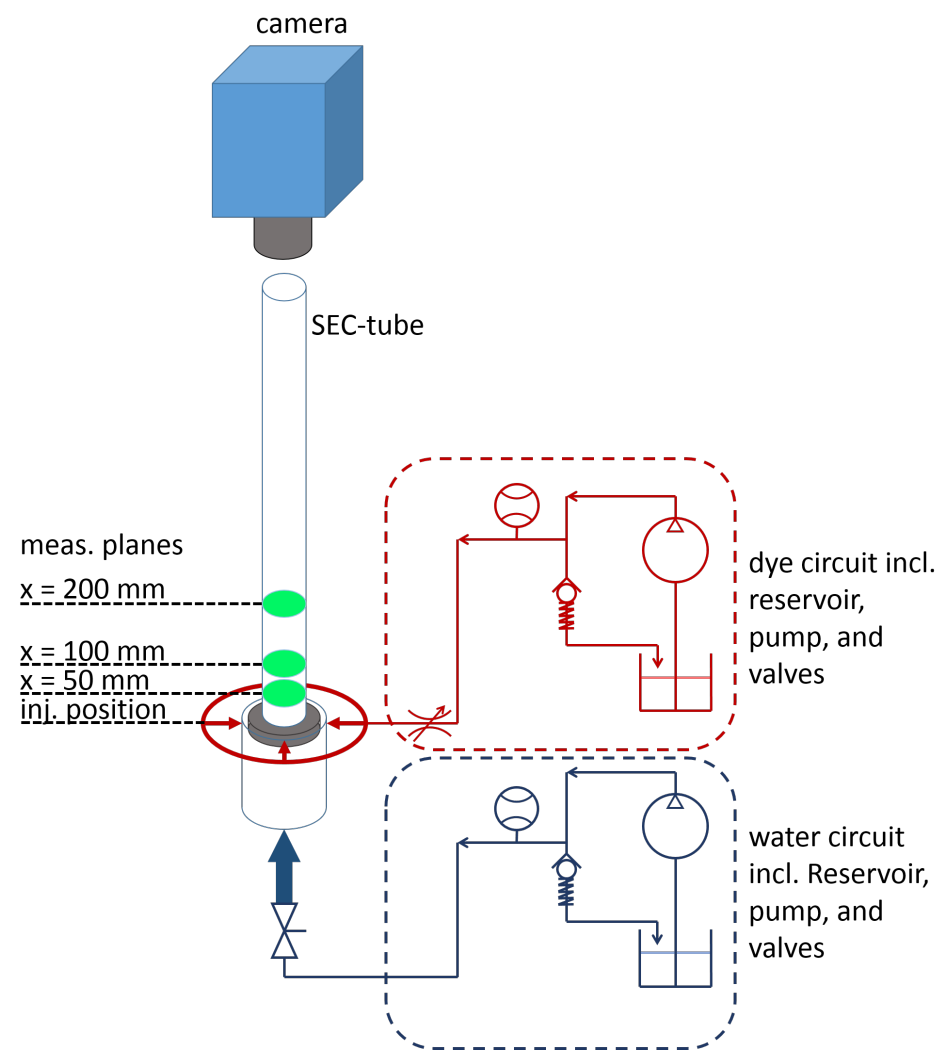

Fig. 2: Sketch of the test-rig.

increases the inner diameter up to the mentioned $40 \mathrm{~mm}$ of the main tube (see Fig. 3). It was assured that the flow is attached to the surface of the diffuser at all times.

Downstream of the diffuser interchangeable disks represent the fuel, in this case dye, inlet. The disk is colored yellow for a better visibility in Fig. 3. Pictures and details of the investigated geometries can be found later in this section. The flow rate of the dye can be adjusted using an electrically driven proportional valve, which was installed upstream of the inlet disc plenum.

Two separated water circuits were realized for the injection of the main and the dye flow. Both circuits are equipped with pressure driven one-way valves in the circuit to generate the needed pressure upstream of the two main valves.

To investigate the mixing quality and determine the unmixedness parameters of the different injection geometries, Planar Laser Induced Fluorenscence (PLIF) was employed in three radial planes at different axial positions downstream of the dye injection. The images were taken with a high-speed camera 


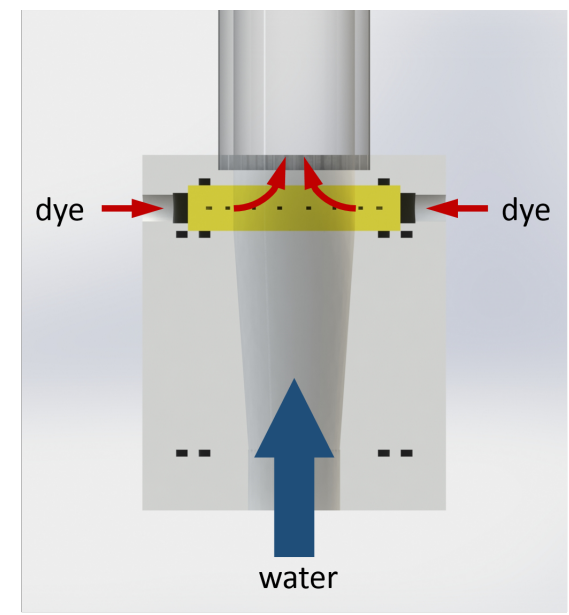

Fig. 3: Cut through the fuel inlet section of the water test-rig.

from the downstream side of the tube. A shutter frequency of $500 \mathrm{~Hz}$ minimizes the blurring of the image due to long exposure times.

21 different injection geometries including round, rectangular, and spatially oscillating (fluidic) jets, as well as slits were investigated. Different cross-section areas were employed to create a broad range of jet in crossflow momentums for all injector disks as well as different frequencies for the fluidic oscillators. The jet in crossflow momentum $J$ is defined as the squared ratio between the jet flow velocity $w_{j}$ and the main flow velocity $w_{0}$.

$$
J=\left(\frac{w_{j}}{w_{0}}\right)^{2}
$$

Note that for all four different injector geometries (i.e., round, rectangle, oscillating, slit) different sizes and amounts of injection ports were tested. This was done to investigate the behavior of these injectors over a wide range of jet in crossflow momentums. Since the volume flow range for all configurations was approximately the same, the different sizes result in different ranges of $J$. This broad range is needed in the SEC process due to the fact that the fuel mass flow is changed within each injection cycle, as mentioned in the introduction. Additionally for distinct injection ports two different numbers of ports were investigated. A summary of the different geometries, including the main flow parameters, can be found in Tab. 1. The Reynolds number of the main flow was $R e=31280$ and thus, a fully turbulent flow was assured. To give an idea of the different injection geometries a three-dimensional model of four of the used injector disks is shown in Fig. 4. For better visibility of the fluidic oscillator, a cut was made through the material to show the geometry.

The investigated spatially oscillating jets were created using fluidic oscillators. From literature [11], it is known that these devices can enhance the spa- 
Table 1: Investigated injector geometries including the main parameters. All units in $\mathrm{mm}$ or $\mathrm{mm}^{2}$ respectively.

\begin{tabular}{llllrlrrr}
\hline \multicolumn{2}{l}{ Disk Outlet geom. } & Outlets Type & \multicolumn{2}{c}{$d_{h}$} & $\mathrm{~A}_{\text {Outlet }}$ & $R e_{\text {jet } \text { min }}$ & $R e_{\text {jet; } \text { max }}$ & $J$ \\
\hline 1 & round & 13 & hole & 1.40 & 20.01 & 4479 & 11143 & $16.7-103.6$ \\
2 & round & 13 & hole & 1.90 & 36.86 & 3542 & 8371 & $5.7-34.2$ \\
3 & round & 13 & hole & 2.40 & 58.81 & 3059 & 7519 & $2.7-16.0$ \\
4 & round & 7 & hole & 2.00 & 21.99 & 6249 & 14485 & $13.2-82.4$ \\
5 & round & 7 & hole & 2.60 & 37.17 & 4916 & 11361 & $5.8-31.2$ \\
6 & round & 7 & hole & 3.30 & 59.87 & 3787 & 9984 & $2.2-15.0$ \\
\hline 7 & rectangle & 13 & hole & 1.26 & 16.33 & 3909 & 9493 & $15.8-93.4$ \\
8 & rectangle & 13 & hole & 1.69 & 21.99 & 3359 & 7138 & $6.4-32.6$ \\
9 & rectangle & 13 & hole & 2.00 & 26.06 & 2548 & 5732 & $2.6-13.4$ \\
10 & rectangle & 7 & hole & 1.73 & 12.09 & 5574 & 12668 & $14.1-87.9$ \\
11 & rectangle & 7 & hole & 2.29 & 16.06 & 4183 & 9983 & $4.5-31.0$ \\
12 & rectangle & 7 & hole & 2.71 & 18.97 & 3308 & 8127 & $2.4-14.7$ \\
\hline 13 & rectangle & 13 & fluidic osc. 1.31 & 16.97 & 3430 & 8092 & $11.2-62.5$ \\
14 & rectangle & 13 & fluidic osc. 1.78 & 23.18 & 2943 & 7223 & $4.4-26.7$ \\
15 & rectangle & 13 & fluidic osc. 2.17 & 28.24 & 1889 & 5618 & $1.2-10.9$ \\
16 & rectangle & 7 & fluidic osc. 1.79 & 12.54 & 4098 & 11088 & $8.6-62.6$ \\
17 & rectangle & 7 & fluidic osc. 2.40 & 16.83 & 3303 & 9726 & $3.1-26.7$ \\
18 & rectangle & 7 & fluidic osc. 2.87 & 20.09 & 2516 & 7828 & $1.3-12.2$ \\
\hline 19 & slit & 1 & - & 0.40 & 50.19 & 893 & 2728 & $8.2-76.3$ \\
20 & slit & 1 & - & 0.60 & 75.22 & 867 & 2701 & $3.4-33.3$ \\
21 & slit & 1 & - & 0.80 & 100.21 & 891 & 2674 & $2.0-18.4$ \\
\hline & & & & & & &
\end{tabular}

tial mixing, especially close to the injection position. The oscillatores used were described in detail in [14]. The oscillatory parameters of these devices were determined by numerical simulations, which were carried out with a validated numerical model [15]. The oscillation frequency was calculated from a time history plot of the velocity inside the mixing chamber.

For each of these 21 configurations, 8 fuel flow rates were investigated at the mentioned three axial positions. Recording 1632 snapshots for each of these tests over 822,000 pictures were taken in total. Each picture was corrected for background reflections and normalized using a homogeneous picture with the maximum dye concentration. It was assured that the dye concentration was low enough to be well within the linear regime of the fluorescence intensity. From the normalized images containing pixel values between 0 and 1, the two unmixedness paramters $U_{x}$ and $U_{t}$ were calculated, which represent the spatial and the temporal mixing quality repectively. Based on the work of Danckwerts [16], the parameters are defined as follows:

$$
U_{x}=\frac{\sigma_{x}^{2}}{\sigma_{0}^{2}}=\frac{\sigma_{x}^{2}}{C_{\infty}^{*}\left(1-C_{\infty}^{*}\right)},
$$




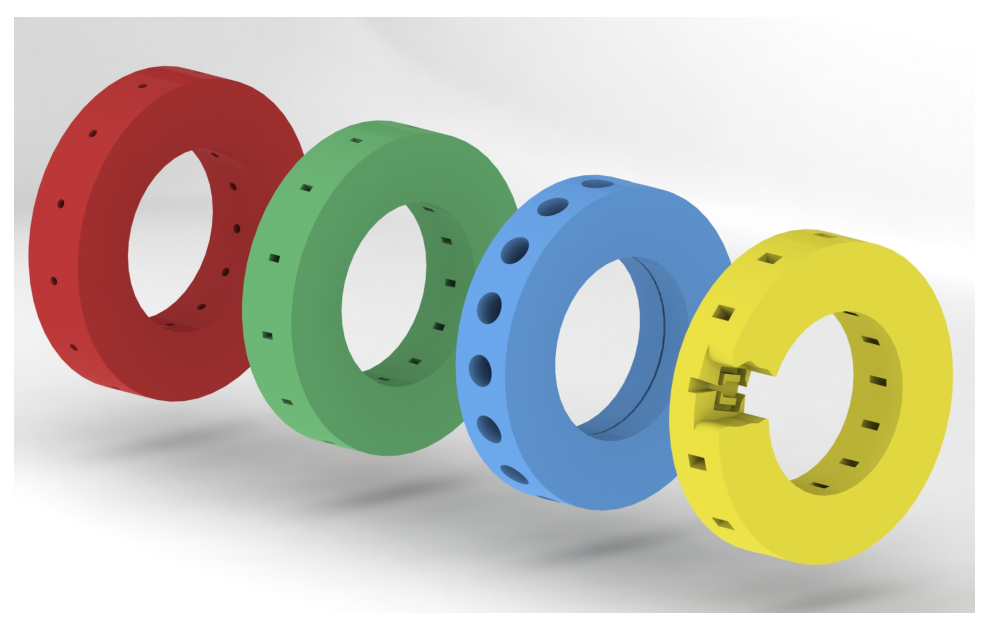

Fig. 4: Colored CAD-Models of the four different injector types (from left to right): round holes (red), rectangular holes (green), slit (blue), and fluidic oscillator (yellow).

$$
\text { where } \quad \sigma_{x}^{2}=\frac{1}{N_{i}-1} \sum_{i=1}^{N_{i}}\left(\overline{C^{*}}(i)-C_{\infty}^{*}\right)^{2},
$$

and $\sigma_{x}^{2}$ denotes the mixture variance of the temporally averaged concentration field $\overline{C^{*}}(i)$, which is recorded by the $N_{i}$ camera pixels and is defined in Eq. 3. The variance immediately before the start of the mixing process $\left(\sigma_{0}^{2}\right)$ is calculated from the dye concentration $C_{\infty}^{*}$. The spatial unmixedness parameter $U_{x}$ represents the average spatial mixing quality and gives a value between 0 (perfectly mixed) and 1 (not mixed at all). To investigate the temporal unmixedness, the parameter $U_{t}$ is used, which employs the variance of all the concentration records. It is defined as:

$$
\begin{aligned}
U_{t} & =\frac{\sigma_{t}^{2}}{\sigma_{0}^{2}}=\frac{\sigma_{t}^{2}}{C_{\infty}^{*}\left(1-C_{\infty}^{*}\right)}, \\
\text { where } \quad \sigma_{t}^{2} & =\frac{1}{N_{i} N_{t}-1} \sum_{i=1}^{N_{i}} \sum_{i=1}^{N_{t}}\left(C^{*}(i, t)-C_{\infty}^{*}\right)^{2} .
\end{aligned}
$$

$N_{t}$ is the number of snapshots recorded during one measurement sequence (1632 in this work). According to Eq. 5, $U_{t}$ captures both spatial and temporal fluctuations in the concentration. These unmixedness parameters can be used as a first measure to evaluate the mixing quality of a given injector geometry.

For the transfer of the experimental results to the numerical investigations, an additional analysis method was employed, which is presented in a next section. 


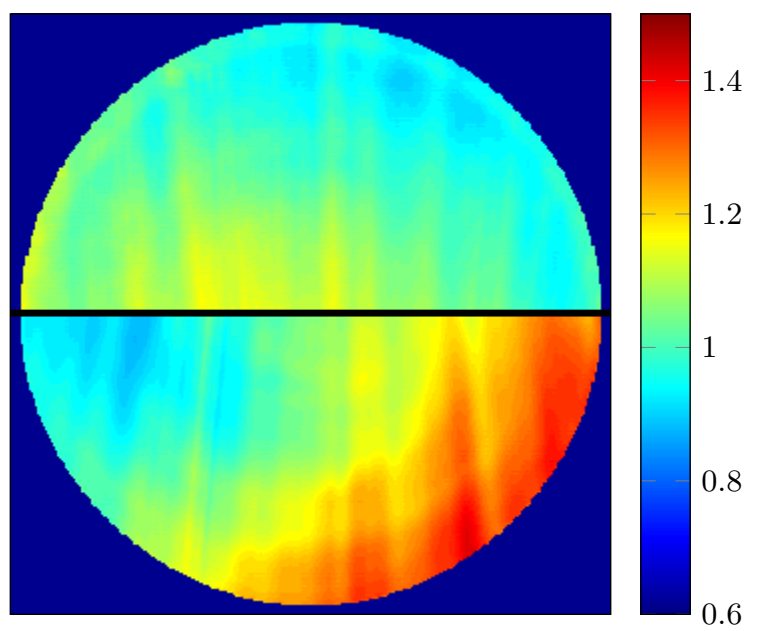

Fig. 5: Averaged, normalized spatial concentration field for disk 13 (upper half) and disk 1 (lower half).

\section{Experimental Results}

As a first impression of the results of the experiments, two pictures of the averaged and normalized concentration field $C$ in the first measurement plane are shown in Fig. 5. For a better visibility, only one half of the pictures is shown for the two different configurations, whereby one of the two pictures was flipped upside-down allowing them to be plotted in one figure. The top half of the picture corresponds to disk 13 (see Tab. 1), an example for a high mixing quality. The lower half of the picture corresponds to disk 1 which shows a rather poor mixing quality due to the different injection geometry. This is clearly visible in the inhomogeneities of the concentration field. In addition to the pictures the concentration histograms for the two measurements are shown in Fig. 6. It is visible that the distribution of the pixel counts has a much sharper peak for disk 13 (see Fig. 6a) than for disk 1 (Fig. 6b). The vast amount of data (see Tab. 1) demands the selection of the injection geometries for further investigations by making use of the defined umixedness parameters. For the SEC-process, a fast, reliable and high-quality mixing is necessary in the radial plane, while almost no mixing is desired in the axial direction. From the steady injection point of view, the latter is unquantifiable since no changes in the dye volume flow are present in the current work. Accordingly, the spatial unmixedness parameter $U_{x}$ is used to investigate the overall mixing parameters of the different geometries.

The range of the spatial unmixedness parameter for the different injection geometries is plotted in Fig. 7 for the first measurement plane $50 \mathrm{~mm}$ downstream of the injection point (see Fig. 2). For this plot, all the geometries of the same type are grouped together to show the overall performance of such a configuration, since the different injection parameters make a plot of the individual 


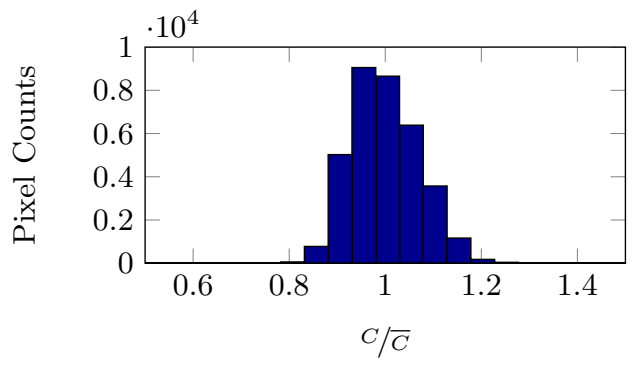

(a) Disk 13 - fluidic osc. $d_{h}=1.31 \mathrm{~mm}$

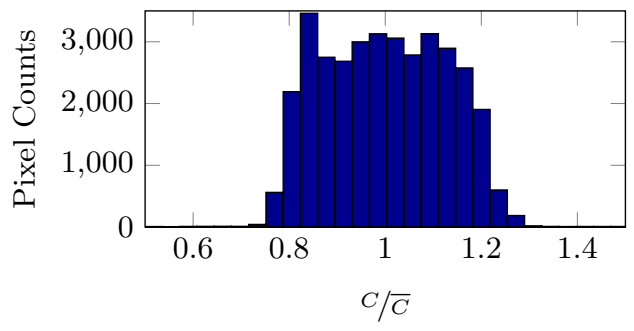

(b) Disk 1 - holes; $d_{h}=1.4 \mathrm{~mm}$

Fig. 6: Histogram of normalized concentration for (a) disk 13 and (b) disk 1 at $x=50 \mathrm{~mm}$.

measurements rather unclear. Accordingly, disks $1-6$ are summed up in the black range, $7-12$ in the red, $13-18$ in the green, and finally the slits $(19-21)$ in the blue range. As one can see in this plot the slits and the round holes give a lower mixing quality than the rectangular holes and the fluidic oscillators. The rectangular holes show a better mixing quality (lower unmixedness parameter) on the lower side of the range than the fluidic oscillators but a worse mixing quality than the fluidic oscillators for the upper boundary of the range. Even though the mixing quality is slightly better for the rectangular holes in some cases, they fail for other injection situations. However, to assure the reliability of the mixing system over a wide range of equivalence ratios, fuel mixtures, and power levels, the fluidic oscillators show a more desirable behavior. Due to these advantages, the fluidic oscillators are chosen to be analyzed in more detail in this work. To gain a deeper insight into the mixing mechanisms of the oscillating jet, two different sizes of fluidic oscillators (disks $13-15$ and disks $16-18$ ) have been designed. Since the range of flow rates is defined by the dye pump, the two different oscillators were manufactured with 3 different aspect ratios each. From literature, it is known that the frequency of these oscillators scales linearly with the volumetric flow rate $[14,17]$. Accordingly, the dependency of the frequency on the jet in crossflow momentum ratio $J$ is of quadratic nature. To show the frequency ranges, $J$ is plotted against the frequency in Fig. 8. It is clearly visible that even though the jet in crossflow momentum $J$ is in the same range for both 


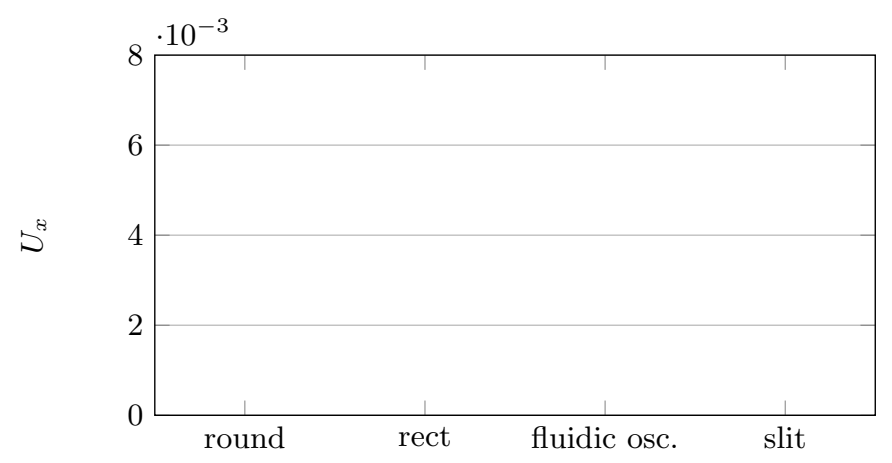

Fig. 7: Spatial unmixedness parameter $U_{x}$ at measurement plane $x=50 \mathrm{~mm}$ for the four diffferent geometries: round jet, rectangular jet, fluidic oscillator, and slit).

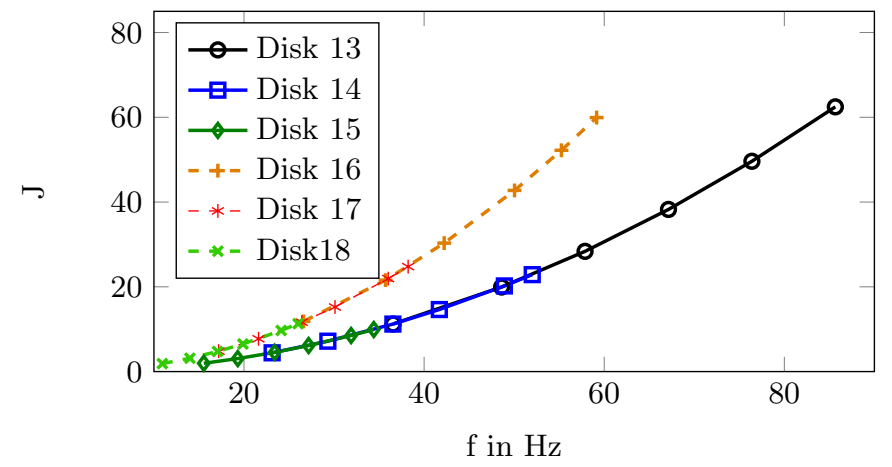

Fig. 8: Frequency of the fluidic oscillators for the different geometries and different values of $J$.

sizes, the oscillation frequency is different. Disks $16-18$, which consist of seven larger fluidic devices give a lower frequency range than disks $13-15$.

Keeping this in mind, the mixing performances of disks 13 and 16 were compared. For the same $J$ and, thus, the same volume flow, Reynolds number, and jet penetration, they create different frequencies. To see the impact of this change in injection geometry, the spatial unmixedness is plotted against $J$ in Fig. 9. Two main conclusions can be made from these results. First, the mixing quality of the smaller oscillators with a higher frequency is better close to the point of injection. This might also be affected by the fact that for the smaller fluidic oscillators, 13 oscillating jets are present, while only 7 were employed for disk 16 . However, it can be stated that a higher oscillation frequency may be favorable for a faster mixing process. The second observation from Fig. 9 is that the mixing quality of both configurations converge as the mixture is convected downstream. 


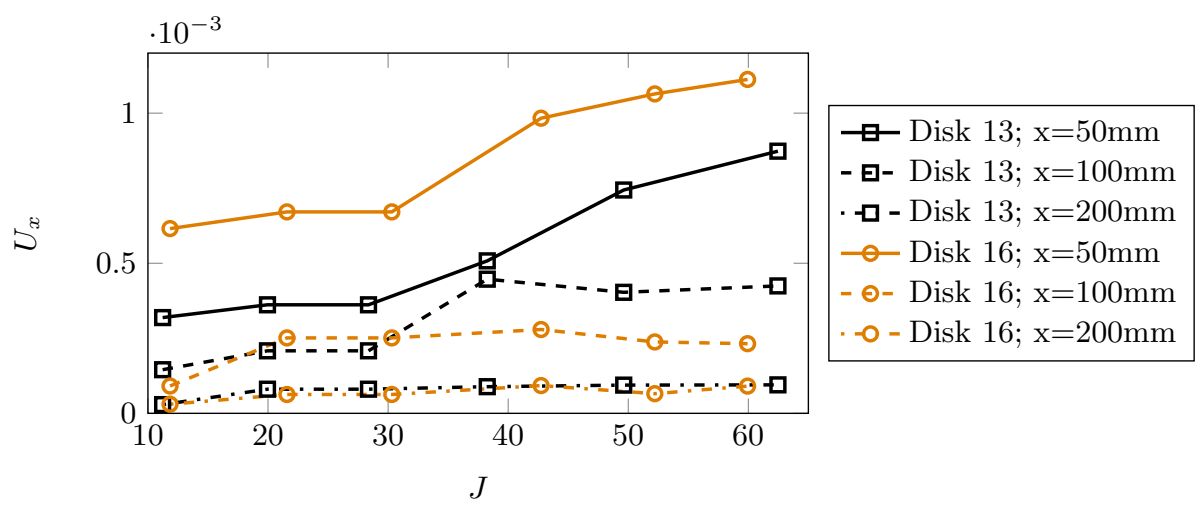

Fig. 9: Spatial unmixedness versus $J$ for disks 13 and 16 for the three measurement planes.

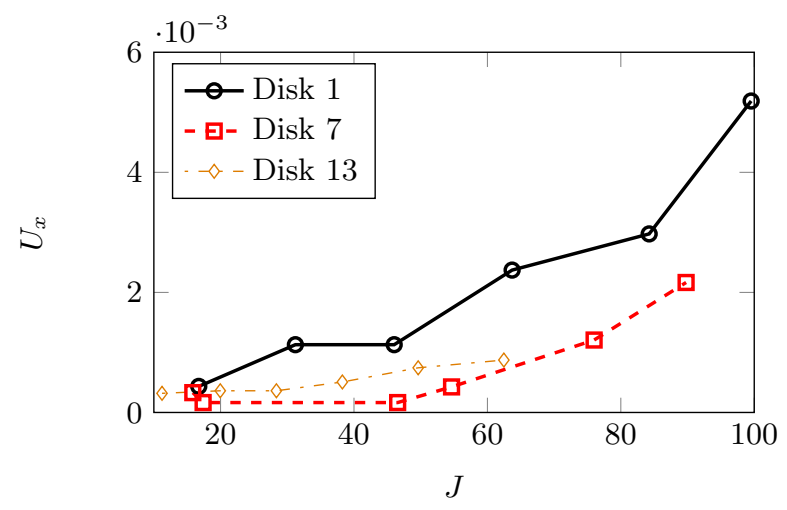

Fig. 10: Spatial unmixedness versus $J$ for disks 1,7 , and 13 at $x=50 \mathrm{~mm}$.

This confirms the findings of Lacarelle et al. [11] that the mixing enhancement of the fluidic oscillators is most pronounced close to the injection plane.

Now, knowing the influence of the oscillating frequency, the influence of the jet in crossflow momentum can be analyzed. To do so the spatial unmixedness parameters of disks 1, 7, and 13 are plotted with respect to $J$ in Fig. 10. These disks have been selected because they have a similar hydraulic diameter and represent three different injection geometries. As already mentioned, the injection geometry for the SEC-process needs to be as independent from the jet in crossflow momentum as possible. From Fig. 10, it can be deduced that this desired characteristic is best represented by the fluidic oscillators (disk 13). Even though the rectangular holes (disk 7 ) have a higher mixing quality for some values of $J$, they show a very pronounced dependency on the jet in crossflow momentum. An even stronger dependency can be seen for the circular holes of disk 1. Keeping in mind that the frequency increases with $J$ and that a higher frequency leads to 


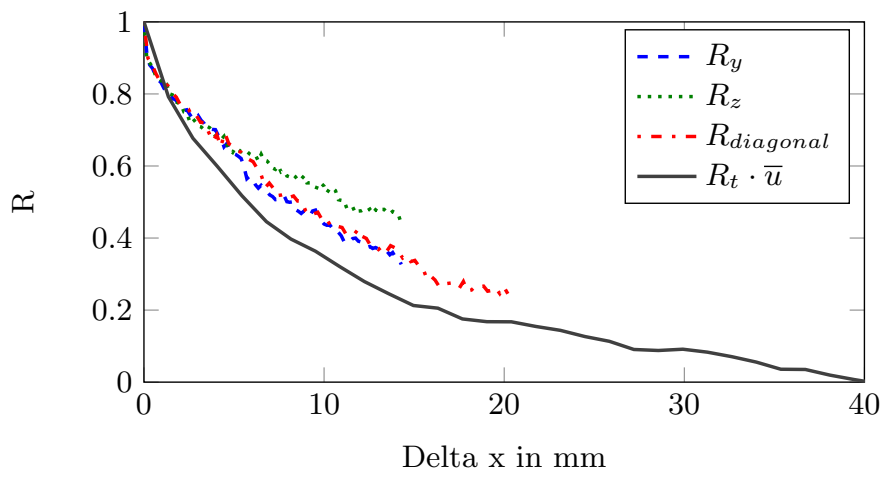

Fig. 11: Correlation coefficients for three spatial directions plus the axial direction calculated using the Taylor hypothesis.

better mixing, the independence of the fluidic injection from $J$ is likely to stem from the increase in the oscillating frequency. These findings again confirm the results from Lacarelle et al. [11], who stated that fluidic oscillators may not only enhance the passive scalar mixing between two fluids but also make that mixing quality less dependent from the jet in crossflow momentum.

Following these observations, disk 13 is the best injection configuration of the investigated geometries. In addition to the mixing quality of the different geometries, the mixing parameters of the best configuration were included in a numerical model of the SEC process to investigate if the mixing quality of the given setup is good enough to assure a reliable Shockless Explosion Combustion.

The data of most promising configuration (injection disk 13) was selected to be investigated by the numerical simulations. Since the most upstream autoignition location (i.e., location of the combustible mixture) in the combustion tube is at least located a distinct distance downstream of the injection point, the data in the third measurement plane $(x=200 \mathrm{~mm})$ was analyzed. From the temporally averaged picture, the spatial variance was calculated according toEq. 3. Since no data was available in the axial direction, except the individually taken radial planes, the Taylor hypothesis was employed to gain an insight into the size of the axial mixing structures of the flow.

To use the Taylor hypothesis, defined as

$$
\frac{\partial C}{\partial t}=-\left.u \frac{\partial C}{\partial x}\right|_{x=x_{0}},
$$

which states that spatial fluctuations in an homogeneously turbulent field, where the streamwise turbulent fluctuations are low $\left(u^{\prime} \ll \bar{u}\right)$, can be calculated from temporal fluctuations at a given coordinate, using the mean streamwise velocity $\bar{u}$. To assure the homogeneous character of the fluctuations in the scalar mixing field, the temporal and spatial correlations where compared. Since all of the correlation curves are reasonably close to each other (see Fig. 11), the use of 
the Taylor hypothesis is justified. Accordingly, from the individual snapshots of concentration at different times in the measurement plane, an integral length scale of the mixing fluctuations was calculated as

$$
\Lambda=\int_{0}^{\Delta x^{*}} R(\Delta x) d(\Delta x),
$$

where $\Delta x^{*}$ is defined as the first intersection of $R(\Delta x)$ and the x-axis. This length scale of $9.7 \mathrm{~mm}$ was then used together with the spatial variance to create an equivalence ratio stratification where the worst possible mixing deviations were imposed. This stratification was then used in the numerical simulation to assess the reliability of the SEC-process.

\section{Numerical Investigation}

The effect of Gaussian noise with the observed standard deviation from the experiment, $\sigma=2.424 \cdot 10^{-3}$, on the homogeneous ignition of a stratified mixture has been numerically investigated by the simulation of the dynamics of a mixture with perturbed equivalence ratios.

The exact numerical setup is based on the internal state of a pipe in which the SEC process is about to enter the combustion stage; prior to this state, gas flowed from the pipe's inlet at $x=0$ to a position $x$ in a time $\tau_{\text {flow }}$ that is assumed to be given via a constant inflow velocity $u_{0}$. This, in turn, is given by the geometry of the pipe and fuel properties or can be arbitrarily chosen for single shot ignitions, respectively. Here, it is chosen such that the gas can flow $40 \mathrm{~cm}$ within the range of the ignition delay times. Since the ignition shall occur homogeneously, the ignition delay time of the gas that ends up at position $x$ must be chosen to be $\tau_{0}+\tau_{\text {flow }}$, such that the remaining ignition delay time $\tau$ at the beginning of the simulation is $\tau_{0}$ everywhere. This choice is done by altering the equivalence ratio $\Phi$ between stoichiometric (1.0) and lean (0.5) values. In the numerical setup, perfect advection without diffusion is assumed, such that the initial values can be calculated by selecting $\Phi$ such that $\tau=\tau_{0}+\tau_{\text {flow }}$ and then solving the mixture in an isochoric 0D reactor for $\tau_{\text {flow }}+\eta \tau_{0}$, where $\eta$ is some fraction smaller than unity and chosen such that the assumption that the fluid is only advected is not violated due to the pressure rise of the reaction. For this simulation, $\eta=0.7$ was chosen.

For the chemical kinetics, the latest fuel suggestion and associated reaction mechanism for a 3 bar test rig, which will also be presented at AFCC 2014 [18], namely a composition of

$$
\mathrm{CH}_{3} \mathrm{OCH}_{3}+1.1 \mathrm{H}_{2}+0.8 \mathrm{CH}_{4}
$$

in air, has been used. The initial temperature was chosen to be $787.5 \mathrm{~K}$.

Within the setup described above, the choices of $\Phi$ were perturbed with different noise levels on equidistant points with $9.7 \mathrm{~mm}$ spacing and linear interpolation in between. The resulting stratification was then placed into a $1 \mathrm{D}$ 
domain as initial conditions for a fluid dynamic simulation that solves the Euler equations with a thermally perfect equation of state and the above reaction mechanism. The in-house code is based on a second-order MUSCL extension of the HLL approximate Riemann solver [19] and uses Strang splitting to couple with an isochoric 0D kinetics code. Reflecting wall boundary conditions have been employed for the left boundary, and a fixed pressure plenum at 3 bar for the right one.

If the full range of equivalence ratios is used and an extremal (deterministic) noise of alternating $3 \sigma$ and $-3 \sigma$ is applied, the different sensitivities at the extremal $\Phi$ values are too high and a detonation develops near $\Phi=1$, traveling towards the $\Phi=0.5$ boundary. The current quality of mixing is, therefore, insufficient and requires improvement. To assess the possibilities with the current mixing and estimate the required variation to allow usage of the full $\Phi$ range, further simulations were conducted. They show two possible ways to achieve homogeneous combustion:

By limiting the choice of $\Phi$ to either $0.5-0.75$ or $0.75-1.0$, the sensitivity is sufficiently reduced to ensure homogeneous combustion. Test case 1 in Fig. 12 shows the simulation results for the upper range. From the ignition delay plot, one can observe that the ignition takes place throughout the domain in a $0.1 \mathrm{~ms}$ window. In the heat map, the transition from blue to yellow/orange is the combustion. The steeper lines are pressure waves in the burnt gas. The results for the lower range of $\Phi$ values are slightly better, with the ignition time varying by only half the upper range's value, but otherwise similar.

Reducing the variance by a factor of $\frac{2}{3}$ is mathematically equivalent to a reduction of the confidence interval to a $2 \sigma$ environment. A simulation with reduced variance can, therefore, be equivalently interpreted as being an improved mixing quality or accepting $5 \%$ non-SEC ignitions. In both cases, the combustion is almost completely homogeneous, as can be seen in test case 2 of Fig. 12 .

\section{Conclusion}

The innovative constant volume combuston process SEC has very high and specific demands on the fuel-air mixing. In order to create the desired levels of mixing quality, several geometries were investigated in a water test-rig. They were analyzed employing planar laser induced fluorescence and evaluated by the unmixedness parameter $U_{x}$.

It was shown that spatially oscillating jets in crossflow created by fluidic oscillators are able to get close to the needed mixing characteristics. In contrast to a slit injection, the round, or the rectangular jet in crossflow, fluidic oscillators allow the creation of a very high mixing quality already close to the fuel injection plane. In addition, the mixing quality is independent from the jet in crossflow momentum for these types of injectors. The results indicate that this is likely to stem from the increasing oscillation frequency of the fluidic oscillators. A higher jet in crossflow momentum leads to a higher volumetric flow in the oscillator and, thus, a higher oscillating frequency. By comparing two different sizes of 

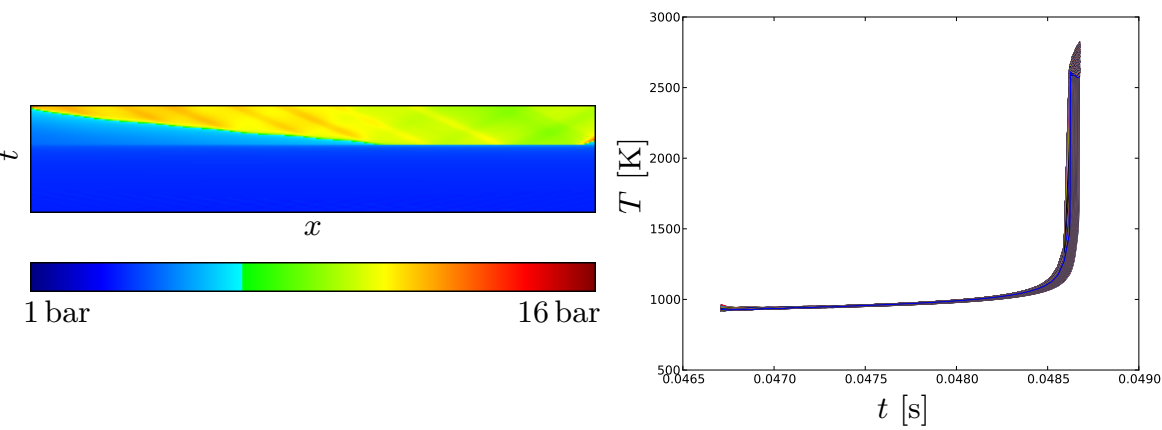

(a) Testcase 1: $\Phi$ limited to $0.75-1.0$.
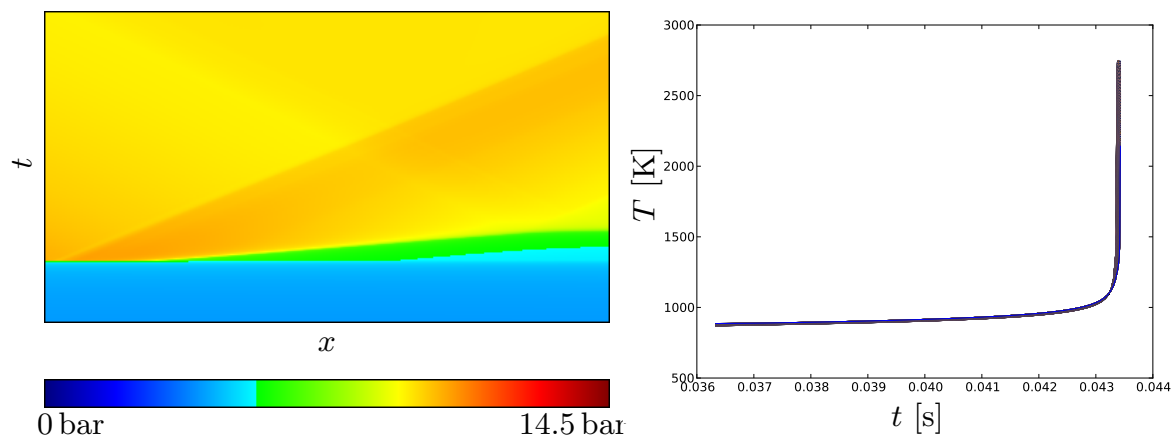

(b) Testcase 2: Noise reduced to $\pm 2 \sigma$ perturbations.

Fig. 12: Ignitions for the different simulations. The heatmaps show pressure evolution, the other plot shows the temperature as a function of time overlain for each of the computational cells.

fluidic oscillators, it was possible to show that higher frequencies are favorable for the mixing process. However, since higher frequencies lead to smaller geometries for the fluidic oscillators the pressure loss of the fuel injection system will increase and might contradict the advantages of a higher mixing quality. Hence, the optimal oscillating frequency must to be identified for every new injection configuration.

In order to validate the achieved mixing quality in the highly demanding SEC-process, a numerical simulation was conducted. Based on these calculations it was possible to show that for an ideal filling charge of the SEC-combustor, the identified perturbations in the equivalence ratio are likely low enough to assure a reliable homogeneous auto-ignition. The experimental verification of these findings and the further investigation of the injection and ignition process 
will be the scope of future work. However, the presented work gives confidence that the proposed SEC-process can be achieved reliably and efficiently.

\section{Acknowledgement}

The authors gratefully acknowledge support by the Deutsche Forschungsgemeinschaft (DFG) as part of collaborative research center SFB 1029 "Substantial efficiency increase in gas turbines through direct use of coupled unsteady combustion and flow dynamics". In addition, the authors would like to thank the CONFET for assistance in the lab and helpful discussions.

\section{References}

1. Keffer, J., Baines, W.: The round turbulent jet in a cross-wind. Journal of Fluid Mechanics 15(4) (1963) 481-496

2. Kamotani, Y., Greber, I.: Experiments on a turbulent jet in a cross flow. AIAA journal 10(11) (1972) 1425-1429

3. Andreopoulos, J.: On the structure of jets in a crossflow. Journal of Fluid Mechanics 157 (1985) 163-197

4. Smith, S.H., Mungal, M.G.: Mixing, structure and scaling of the jet in crossflow. Journal of Fluid Mechanics 357 (February 1998) 83-122

5. Eroglu, A., Breidenthal, R.: Structure, penetration, and mixing of pulsed jets in crossflow. AIAA journal 39(3) (2001)

6. Johari, H., Pacheco-Tougas, M., Hermanson, J.: Penetration and Mixing of Fully Modulated Turbulent Jets in Crossflow. AIAA Journal 37(7) (July 1999) 842-850

7. Johari, H.: Scaling of Fully Pulsed Jets in Crossflow. AIAA Journal 44(11) (November 2006) 2719-2725

8. Weston, R., Thames, F.: Properties of aspect-ratio- 4.0 rectangular jets in a subsonic crossflow. Journal of Aircraft 16(10) (1979) 701-707

9. Plesniak, M.W., Cusano, D.M.: Scalar mixing in a confined rectangular jet in crossflow. Journal of Fluid Mechanics 524 (February 2005) 1-45

10. Nathan, G., Mi, J., Alwahabi, Z., Newbold, G., Nobes, D.: Impacts of a jet's exit flow pattern on mixing and combustion performance. Progress in Energy and Combustion Science 32(5-6) (September 2006) 496-538

11. Lacarelle, A., Paschereit, C.O.: Increasing the Passive Scalar Mixing Quality of Jets in Crossflow With Fluidics Actuators. Journal of Engineering for Gas Turbines and Power 134(2) (2012) 021503

12. Clarke, A., Gerrard, A., Holliday, L.: Some experiences in gas turbine combustion chamber practice using water flow visualization techniques. Symposium (International) on Combustion 9(1) (1963) 878-891

13. Lacarelle, A., Sebastian, G., Paschereit, C.O.: A Quantitative Link Between ColdFlowscalar Unmixness and NOX Emissions in a Conical Premixed Burner. In: ASME Turbo Expo 2010. Number X (2010) ASME GT2010-23132

14. Bobusch, B.C., Woszidlo, R., Bergada, J.M., Nayeri, C.N., Paschereit, C.O.: Experimental study of the internal flow structures inside a fluidic oscillator. Experiments in Fluids 54(6) (June 2013) 1559 
15. Krüger, O., Bobusch, B.C., Woszidlo, R., Paschereit, C.O.: Numerical Modeling and Validation of the Flow in a Fluidic Oscillator. In: 21st AIAA Computational Fluid Dynamics Conference, Reston, Virginia, American Institute of Aeronautics and Astronautics (June 2013)

16. Danckwerts, P.: The definition and measurement of some characteristics of mixtures. Applied Scientific Research, Section A 3 (1952) 279-296

17. Bobusch, B.C., Woszidlo, R., Krüger, O., Paschereit, C.O.: Numerical Investigations on Geometric Parameters Affecting the Oscillation Properties of a Fluidic Oscillator. Proceedings of the 21st AIAA Computational Fluid Dynamics Conference (2013)

18. Cai, L., Pitsch, H.: Tailoring fuels for shockless explosion engine (preliminary title). Proceedings of AFCC (2014)

19. Harten, A., Lax, P.D., Van Leer, B.: On upstream differencing and godunov-type schemes for hyperbolic conservation laws. SIAM Review (1983) 35-61 